Communications in Physics, Vol. 26, No. 3 (2016), pp. 247-252

DOI:10.15625/0868-3166/26/3/8945

\title{
A HIGH Q-FACTOR, EASY-TO-USE, BROADBAND Ce:LiCAF LASER RESONATOR BASED ON TOTAL INTERNAL REFLECTION
}

\author{
ARITA REN, EMPIZO MELVIN JOHN F, MINAMI YUKI, LUONG VIET MUI, \\ TANIGUCHI TAKAYA, YAMANOI KOHEI, SHIMIZU TOSHIHIKO \\ AND SARUKURA NOBUHIKO \\ Institute of Laser Engineering, Osaka University, 2-6 Yamadaoka, Suita, Osaka 565-0871, Japan \\ RADUBAN MARILOU CADATAL ${ }^{1,2}$ \\ ${ }^{1}$ Institute of Laser Engineering, Osaka University, 2-6 Yamadaoka, Suita, Osaka 565-0871, Japan \\ ${ }^{2}$ Centre for Theoretical Chemistry and Physics, Institute of Natural and Mathematical Sciences, \\ Massey University, Albany, Auckland 0632, New Zealand
}

PHAM VAN DUONG, NGUYEN XUAN TU AND PHAM HONG MINH ${ }^{\dagger}$

Institute of Physics, Vietnam Academy of Science and Technology, 10 Dao Tan, Ba Dinh, Hanoi 10000, Vietnam

${ }^{\dagger}$ E-mail: phminh@iop.vast.ac.vn

Received 29 November 2016

Accepted for publication 05 January 2017

\begin{abstract}
We report a design of a cerium-doped lithium calcium hexafluoro aluminumate

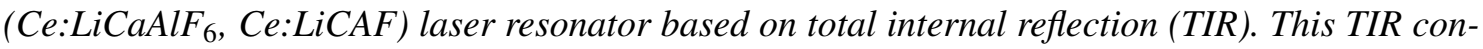
figuration is established by placing the Ce:LiCAF crystal between two calcium fluoride ( $\left.\mathrm{CaF}_{2}\right)$ Pellin-Broca prisms. The laser resonator exhibits a 289-nm emission with 3.5-nm spectral linewidth and 4.4-ns pulse duration. Results show that the present configuration can be a high Q-factor, easy-to-use, broadband prism resonator suitable as a laser oscillator not only in the ultraviolet $(U V)$ region but also down to vacuum ultraviolet $(V U V)$ wavelengths where alignment is restricted to vacuum environments.
\end{abstract}

Keywords: laser resonator, total internal reflection, fluorides, VUV.

Classification numbers: 42.60.Da, 42.60.-v. 


\section{INTRODUCTION}

Many technological applications such as spectroscopy [1] and nanofabrication [2] now require excitation sources with short wavelengths and short pulse durations. Some of the vacuum ultraviolet (VUV) and extreme ultraviolet (EUV) light sources include synchrotron orbital radiation, x-ray free electron lasers (XFELs), and excimer lasers. Synchrotron and XFELs are the ideal light sources, but these systems are large and are difficult to handle. On the other hand, conventional excimer lasers such as krypton $(\mathrm{Kr})$, xenon $(\mathrm{Xe})$, argon $(\mathrm{Ar})$, and fluorine $\left(\mathrm{F}_{2}\right)$ are compact, but they pose safety concerns with their gas contents. Developing compact, easy-touse, and efficient laser systems is not only desirable but also important for many technological applications.

Rare earth-doped fluoride crystals are prominent UV to VUV laser media because of their wide band gaps [3]. Neodymium-doped lanthanum fluoride $\left(\mathrm{Nd}: \mathrm{LaF}_{3}\right)$, for example, has been observed to exhibit lasing at $172 \mathrm{~nm}[4,5]$. In comparison, Nd-doped lanthanum barium fluoride $(\mathrm{Nd}: \mathrm{LaBaF})$ has a broader fluorescence bandwidth than $\mathrm{Nd}: \mathrm{LaF}_{3}$ and has been suggested as a tunable laser material and for short pulse amplification [6]. Furthermore, Nd-doped lutetium lithium fluoride (Nd:LuLiF) can be a potential solid-state VUV laser media like Nd:LaF via twophoton excitation $[7,8]$. Investigating different potential laser media can lead to the development of broadband and efficient solid-state laser systems.

In this regard, we report on the design of a Ce:LiCAF laser resonator based on total internal reflection (TIR). The TIR configuration is established by two calcium fluoride $\left(\mathrm{CaF}_{2}\right)$ Pellin-Broca (PB) prisms. The laser resonator can be used to lase a solid-state laser media in the UV region using a Ce:LiCAF crystal. Our results show that the present resonator configuration can be used not only in the UV region but also down to VUV wavelengths.

\section{EXPERIMENTAL}

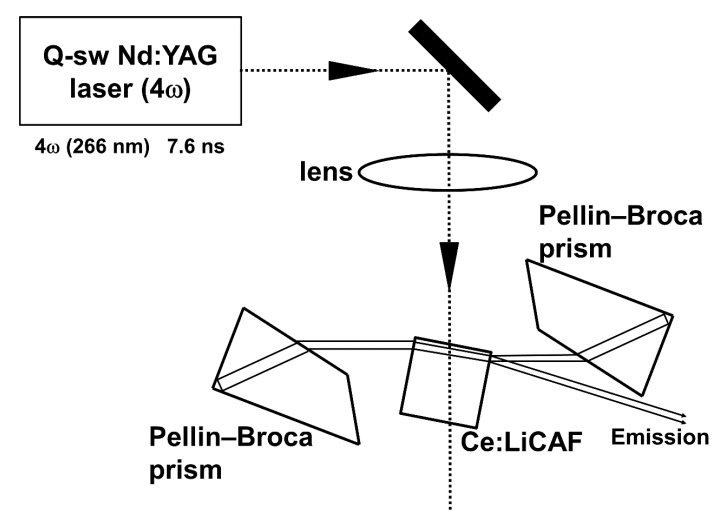

Fig. 1. Schematic diagram of the Ce:LiCAF laser resonator based on total internal reflection. The Ce:LiCAF crystal was pumped using the fourth harmonic $(4 \omega, 266 \mathrm{~nm})$ of a Q-switched Nd:YAG laser.

Figure 1 shows the schematic diagram of the Ce:LiCAF laser resonator based on TIR. The fourth harmonics $(4 \omega, 266 \mathrm{~nm})$ of a Q-switched Nd:YAG laser with $7.6 \mathrm{~ns}$ pulse duration and 
$10 \mathrm{~Hz}$ repetition pulse rate was used as an excitation source. The laser beam was focused on the Ce:LiCAF crystal by a $70 \mathrm{~cm}$ focal-length lens to create a $5 \mathrm{~mm}$ diameter spot, that means the pump spot overlaps the entire length of the laser crystal. The Czochralski method-grown crystal has $5.0 \times 5.0 \times 10 \mathrm{~mm}$ dimensions and has polished surfaces. Two $\mathrm{CaF}_{2}$ Pellin-Broca prisms were used to establish TIR on the side-pumped laser resonator. The Ce:LiCAF crystal was placed between the PB prisms at an angle of $11^{\circ}$ with respect to the oscillation axis. The $\mathrm{CaF}_{2}$ prisms were aligned with respect to the crystal so that the incident angle was around the Brewster angle at $289 \mathrm{~nm}$. One of the PB prisms (left prism in Fig. 1) was rotated by a few degrees to allow a part of the $290 \mathrm{~nm}$ laser emission to escape the resonator. The slope efficiency, spectral profile and pulse duration of the laser emission were evaluated.

\section{RESULTS AND DISCUSSIONS}

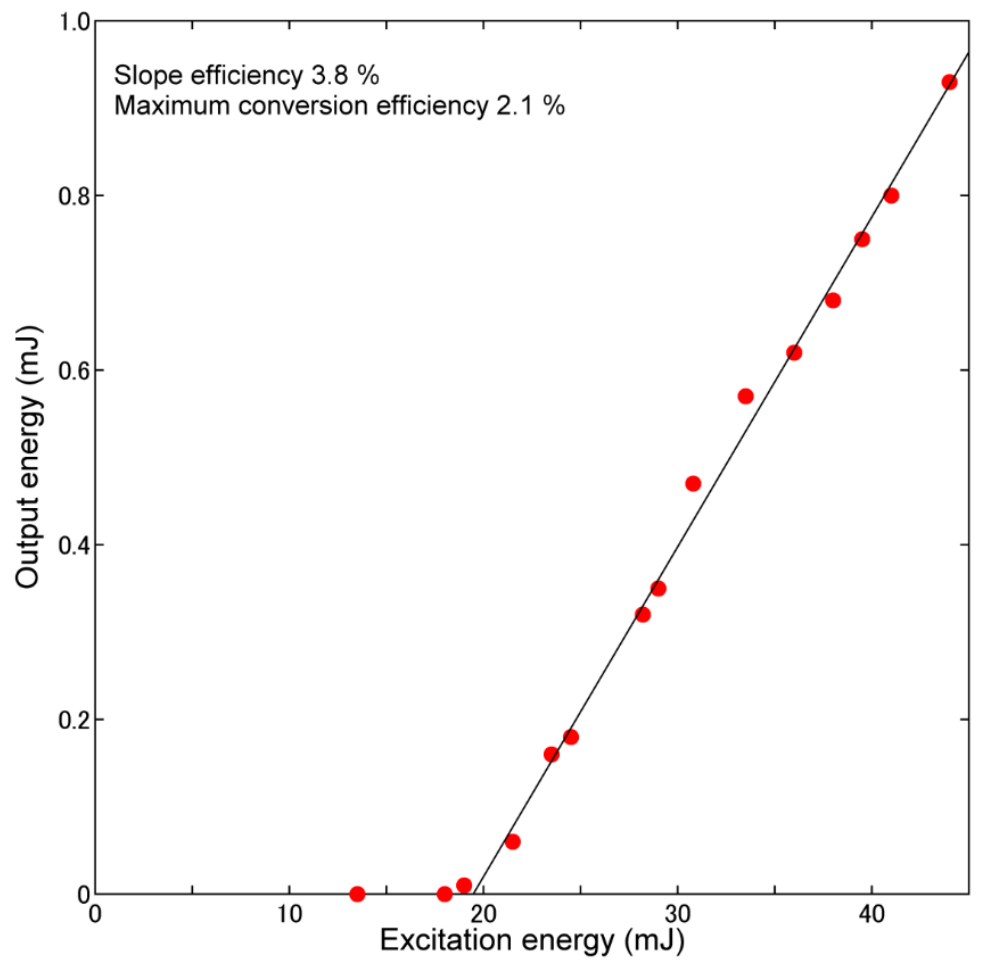

Fig. 2. Output energy of the Ce:LiCAF laser resonator as a function of the Nd:YAG excitation energy. The slope efficiency is around $3.8 \%$, and maximum conversion efficiency is around $2.1 \%$.

Figure 2 shows the output energy of the Ce:LiCAF laser resonator as a function of the $\mathrm{Nd}$ :YAG excitation energy. The slope efficiency is estimated to be around 3.8\%, and the maximum conversion efficiency is around $2.1 \%$. The obtained efficiency of the present prism resonator is lower compared to previous reports. A $46 \%$ conversion efficiency has been obtained from Ce:LiCAF using an output coupler with $60 \%$ reflectance [9]. The lower efficiency can be attributed 
to the low reflectance of the crystal's side surface. Assuming that the refractive index (n) of Ce:LiCAF is the same to that of undoped $\mathrm{LiCaAlF}_{6}(1.404)$ [10], the reflectance will be only $2.7 \%$. The typical absorption coefficient of Ce:LiCAF at the emission wavelength $(290 \mathrm{~nm})$ is $0.2 \mathrm{~cm}^{-1}$ and its absorption coefficient at the pump wavelength $(266 \mathrm{~nm})$ is $3.2 \mathrm{~cm}^{-1}$ [11]. Therefore, the figure of merit of our oscillator setup is about 16. The figure of merit was calculated as the ratio of the absorption coefficient of Ce:LiCAF at the pump and emission wavelengths (3.2 $\left.\mathrm{cm}^{-1} / 0.2 \mathrm{~cm}^{-1}\right)$. The two $\mathrm{CaF}_{2}$ Pellin-Broca prisms are highly transparent at $290 \mathrm{~nm}$, hence its absorption coefficient at the lasing wavelength $(290 \mathrm{~nm})$ is close to zero. Nevertheless, our results demonstrate the design of a laser oscillator based on TIR using two PB prisms. This design will be even more important for VUV lasing where the set-up needs to be in vacuum. Figure 3 (a) and 3(b) show the spectral and temporal profiles of the Ce:LiCAF laser emission. The laser emission peak is centered at $289 \mathrm{~nm}$, and its spectral linewidth is $\sim 3.5 \mathrm{~nm}$. Compared with the $7.6 \mathrm{~ns}$ pulse duration of the pump beam, the laser pulse duration of the PB prisms laser is only $4.4 \mathrm{~ns}$. These results demonstrate that Ce:LiCAF laser emission can be achieved using the present laser resonator.
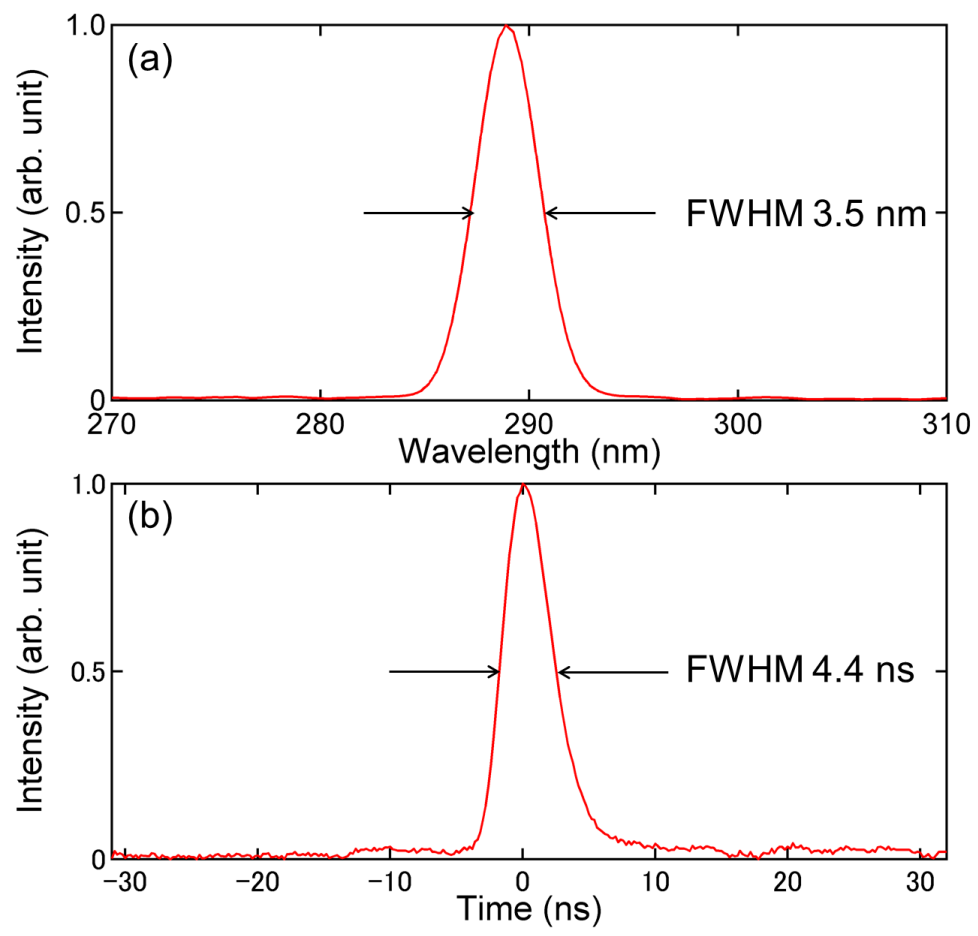

Fig. 3. (a) Spectral profile and (b) temporal profile of the Ce:LiCAF laser resonator emission. The laser resonator has a peak wavelength at $289 \mathrm{~nm}$ with $3.5-\mathrm{nm}$ spectral linewidth and a pulse duration of $4.4 \mathrm{~ns}$.

Laser oscillation can be achieved by TIR using two Pellin-Broca prisms. The PB prisms were used to achieve Brewster angle incidence at the prism surfaces and, therefore, minimize reflection losses of 290-nm laser emission. Moreover, typical laser oscillators consist of dielectric 
multilayer mirrors whose reflectance greatly depends on the incident wavelength. Our results show that alignment of the Pellin-Broca resonator can be varied for different laser media due to the low reflection loss of p-polarized light at angles away from the Brewster angle of the prism. For example, a $2^{\circ}$ difference between the incidence and the Brewster angles causes less than $0.1 \%$ reflection loss [12]. This means that a high Q-factor resonator can be maintained with even a rough alignment of the prisms. Maintaining a high Q-factor resonator is suitable for evaluating whether the candidate laser medium can oscillate or not. Moreover, a $90^{\circ}$-angle prism makes the alignment of the resonator easy because the number of rotation axes, which require precise adjustment of the prisms, is decreased by one. The use of Brewster incidence of the prism also reduces the risk of optical damage compared to using antireflection coating at normal incidence. $\mathrm{CaF}_{2}$ is transparent causes very minimal loss in the VUV region. For instance, internal absorption loss of a $10 \mathrm{~mm}$ thick Nikon $\mathrm{CaF}_{2}$ lens at $157-\mathrm{nm}$ wavelength is less than $0.5 \%$ [13]. For these reasons, the $\mathrm{CaF}_{2}$ Pellin-Broca prism resonator that we have designed can be a high Q-factor, easy-to-use, broadband laser resonator suitable as a laser oscillator in different wavelength regions not only in UV but also down to VUV.

\section{CONCLUSION}

We designed a Ce:LiCaAF laser resonator based on TIR established by using two $\mathrm{CaF}_{2}$ Pellin- Broca prisms. Laser emission from the Ce:LiCAF crystal was observed at around $289 \mathrm{~nm}$. Our results suggest that this prism resonator can potentially be used in the VUV region where alignment of a resonator is limited by vacuum conditions. Further investigations are anticipated in the future to demonstrate VUV lasing with this resonator using other fluoride crystals such as $\mathrm{Nd}: \mathrm{LiCAF}, \mathrm{Nd}: \mathrm{LaF}_{3}, \mathrm{Nd}: \mathrm{LaBaF}$ or Nd:LuLiF.

\section{ACKNOWLEDGMENTS}

This work was supported by the Vietnam National Foundation for Science and Technology Development (NAFOSTED) under Grant Number 103.03-2015.29.

\section{REFERENCES}

[1] D. J. Jones, R. H. French, H. Mullejans, S. Loughin, A. D. Dorneich, and P. F. Carcia, J. Mater. Res. 14 (1999) 4337.

[2] H. Sugimura, L. Hong, and K. H. Lee, Jpn. J. Appl. Phys. 44 (2005) 5185.

[3] K. H. Yang, and J. A. DeLuca, Appl. Phys. Lett. 29 (1976) 499.

[4] R. W. Waynant, and P. H. Klein, Appl. Phys. Lett. 46 (1985) 14.

[5] M. A. Dubinskii, A. C. Cefalas, E. Sarantopoulou, S. M. Spyrou, and C. A. Nicolaides, J. Opt. Soc. Am. B9 (1992) 1148.

[6] M. Cadatal, Y. Furukawa, S. Ono, M. Pham, E. Estacio, T. Nakazato, T. Shimizu, N. Sarukura, K. Fukuda, T. Suyama, A. Yoshikawa, F. Saito, J. Lumin. 129(2009), 1629.

[7] T. Nakazato, M. Cadatal-Raduban, K. Yamanoi, M. Tsuboi, Y. Furukawa, M. Pham, E. Estacio, T. Shimizu, N. Sarukura, K. Fukuda, T. Suyama, T. Yanagida, Y. Yokota, A. Yoshikawa, and F. Saito, IEEE Trans. Nucl. Sci. 57 (2010), 1208.

[8] K. Yamanoi, Y. Minami, R. Nishi, Y. Shinzato, M. Tsuboi, M. V. Luong, T. Nakazato, T. Shimizu, N. Sarukura, M. Cadatal-Raduban, M. H. Pham, H. D. Nguyen, Y. Yokota, A. Yoshikawa, M. Nagasono, T. Ishikawa, Opt. Mater. 35 (2013) 2030.

[9] V. A. Fromzel, and C. R. Prasad: OSA. ASSP. (2013). 
[10] K. Shimamura, H. Sato, A. Bensalah, H. Machida, N. Sarukura, and T. Fukuda, J. Alloys. Compd. 343 (2002) 204.

[11] S. Ono, Y. Suzuki, T. Kozeki, H. Murakami, H. Ohtake, N. Sarukura, H. Sato, S. Machida, K. Shimamura, and T. Fukuda, Appl. Opt. , 41 (2002) 7556-7560.

[12] I. H. Malitson, Appl. Opt. 2 (1963)1103.

[13] Nikon $\mathrm{CaF}_{2}$ NICF series catalogue data: http://www.nikon.com/products/glass/lineup/caf2/pdf/caf2-e.pdf 